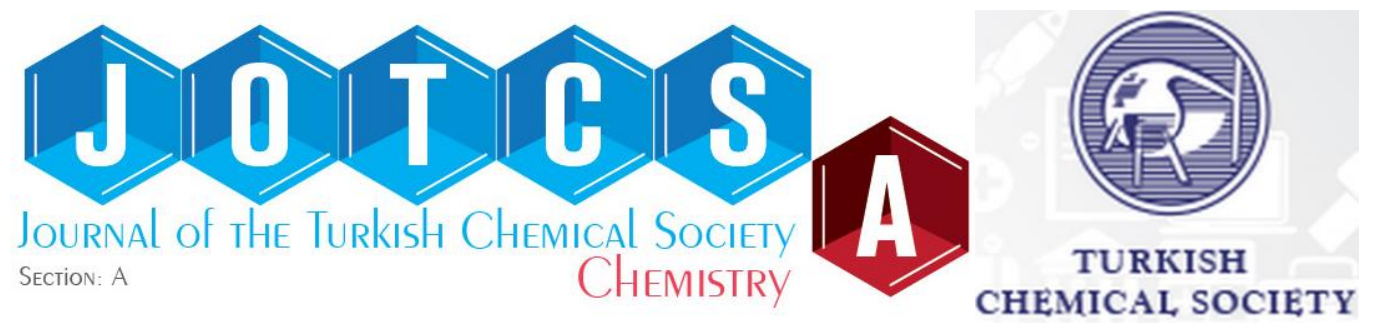

\title{
Simultaneous Deposition of Poly(o-anisidine) and Noble Ag Particles on Wool Fabric and The Evaluation of Its Performance as a Catalyst in Dye Reduction
}

\author{
Meryem Kalkan Erdoğan $\triangle(D)$ and Meral Karakışla* $\Delta$ (D)
}

Department of Chemistry, Faculty of Science, Ankara University, Degol Street, 06100 Tandoğan Ankara/TURKEY

Abstract: The conductive poly(o-anisidine) (POA) and noble Ag particles were simultaneously deposited on wool fabrics, by the polymerization of $O$-anisidine with the oxidant mixtures of silver nitrate and ammonium persulfate in different sulfonic acids media. The effect of concentrations of sulfonic acids was investigated on the contents of conductive components (POA/Ag\%) and volume resistivity of the composites. The change in the surface morphology of POA coating, distribution of Ag particles and conformance of noble Ag particles were identified with SEM, EDX, and XRD techniques, respectively. It was observed that the usage of sulfonic acids significantly increased the coating density of the POA/Ag, compared to a control sample prepared in $1.0 \mathrm{M} \mathrm{HNO}_{3}$ solution. The composites were tested in the catalytic reduction of a dye molecule (methylene blue) with the existence of excess sodium borohydride, and a relatively high catalytic activity $(70 \%)$ was obtained at 75 min compared to a control POA/wool sample.

Keywords: wool fabric, poly(o-anisidine), noble Ag particles, sulfonic acids, catalytic dye reduction.

Submitted: January 13, 2019. Accepted: May 12, 2019.

Cite this: Kalkan Erdoğan M, Karakışla M. Simultaneous Deposition of Poly(o-anisidine) and Noble Ag Particles on Wool Fabric and The Evaluation of Its Performance as a Catalyst in Dye Reduction. JOTCSA. $2019 ; 6(2): 225-36$.

DOI: $\underline{\text { https://dx.doi.org/10.18596/jotcsa.512360. }}$

*Corresponding author. E-mail: meral.karakisla@gmail.com, Tel: +903122126720, Fax: +903122232395 .

\section{INTRODUCTION}

Preparation of fibrous textile composites with conductive polymers such as polyaniline, polypyrrole, polythiophene and their derivatives has been of interest the researchers, because of enabling fabrication of materials to be used in various areas such as electromagnetic interference (EMI) shielding (1-3), heating devices (4), sensor applications ranging from environmental to medical purposes (5-7), wearable electronics (8) and so on (9). Among all wet chemical methods for preparation of these textile composites, the oxidative polymerization is found practical in terms of one-step modification of surfaces with conductive polymers. In a conventional route, a textile material impregnated with conjugated monomer solution is polymerized with conventional oxidant solutions such as ammonium persulfate, anhydrous and/or hydrated iron(III) salts, dichromate salts and silver nitrate depending on the selected polymerization medium. Among them, the usage of silver nitrate as oxidant is quite advantageous in terms of providing simultaneous conductive polymer coating and metallic Ag particles' deposition on textile surfaces through redox reaction of silver nitrate with conjugated monomers $(10,11)$. In this way, the researchers reported that the composites gained multifunctional properties, such as improved EMI shielding, catalytic activity, antibacterial activity and SERS activity $(10,12-$ 14). Although these aforementioned superiorities, the usage of silver nitrate oxidant may have some limitations such as long induction periods extending to several weeks $(15-17)$. The researchers reported to solve this obstruction using an accelerating effect such as UV-light, phenylenediamines and secondary oxidant such 
as ammonium persulfate without formation of unwanted $\mathrm{Ag}$ salts $(12,18-21)$. In this study, we also preferred using a secondary persulfate oxidant.

In our previous study, we demonstrated that the surface of polyester nonwoven could be homogenously decorated with highly conductive POA and Ag particles, by oxidative polymerization of $o$-anisidine with silver nitrate and acceleration with ammonium persulfate in the media of inorganic and sulfonic acids that do not lead to the formation of unwanted $\mathrm{Ag}$ salts while providing desired conductivity (12). In this presented work, we also intended to coat the natural wool fabrics simultaneously and homogenously with POA and Ag particles, in the media of various sulfonic acids such as camphorsulfonic acid, (CSA) p-(toluenesulfonic acid) (PTSA) and dodecylbenzenesulfonic acid (DBSA). Then we examined the usability of these composite fabrics as catalyst in the reduction of a model dye molecule, methylene blue (MB) to leucomethlyene blue (LMB), with the existence of sodium borohydride as a reducing agent. In a very recent study published by our group, the catalytic reduction of $M B$ was investigated by using two-steps of POA and $\mathrm{Ag}$ particles decorated wool composites (22). This presented study is different from our recent published work in terms of many steps. For example, in our previous work we first coated wool fabrics with POA polymer in the medium of $\mathrm{HCl}$, then dedoped it in alkali medium and redoped the composite with different numbers of carboxylic acid containing acids such as acetic, citric and ascorbic acids. The decoration of Ag particles was achieved by these redoping acids that were "subsequently incorporated" to the POA chains on composites. However in our presented work, we simultaneously coated the surface of wool with POA and noble $\mathrm{Ag}$ particles in the media of different sulfonic acids that are "simultaneously (in other words, in-situ) incorporated" to POA chains. We also investigated some properties of the reduced $\mathrm{Ag}$ particles such as changing particle morphology with selected polymerization medium and investigated the effect of selected polymerization medium on catalytic activity of the composites.

\section{EXPERIMENTAL SECTION}

Wool fabrics (Yünsa Wool Industry Trade Co., TURKEY) were used after washing in $4 \mathrm{~g} / \mathrm{L}$ aqueous standard ECE detergent solution (1:50 bath ratio) at $40{ }^{\circ} \mathrm{C}$ for $1 \mathrm{~h}$, and drying under vacuum at $50{ }^{\circ} \mathrm{C}$. $\mathrm{O}$-anisidine (Merck) was used after vacuum distillation. Other chemicals such as ammonium persulfate, silver nitrate, sodium borohydride, $\mathrm{HNO}_{3}, \mathrm{CSA}, \mathrm{PTSA}$, DBSA, methanol, $M B$ were obtained from Sigma-Aldrich and used as received.

\section{Simultaneous Deposition of POA and Noble Ag Particles on Wool}

The simultaneous deposition of POA and $\mathrm{Ag}$ particles on fabrics were performed similarly to our previous study containing preparation of POA/Ag/PET nonwoven composites with some differences (12). Briefly, wool fabrics $2 \mathrm{~cm} \times 2 \mathrm{~cm}$ in sizes were impregnated with $0.2 \mathrm{M} \mathrm{o}$-anisidine solutions prepared in $10 \mathrm{~mL}$ certain concentrations of sulfonic acid solutions for a while. Then $0.1 \mathrm{M}$ of $2 \mathrm{~mL}$ silver nitrate solutions prepared in same sulfonic acid solutions were added drop by drop onto the fabric-containing medium. The initiation step of the polymerization was accelerated with the addition of $0.1 \mathrm{M}$ ammonium persulfate solutions $(3 \mathrm{~mL}$ ) prepared in the selected acid solutions, and the reaction was continued for $3 \mathrm{~h}$ at $20^{\circ} \mathrm{C}$. The composite samples separated from polymerization solutions were adequately washed with distilled water and methanol to remove the residual oxidants and monomer, respectively. The obtained composites were shaken in the selected sulfonic acid solutions for redoping and then dried at $50{ }^{\circ} \mathrm{C}$ under the vacuum. The contents of conductive components of the fabrics, (POA/Ag) (\%) were calculated gravimetrically by discarding the weight of the composite from the weight of untreated wool.

For comparative purposes, POA coated wool fabrics were also prepared. For this case, same process steps including monomer impregnation, polymerization medium selection, and polymerization duration, redoping and drying were followed with the simultaneous deposition of POA and Ag particles except using silver nitrate solution as oxidant. In addition, the ammonium persulfate/o-anisidine mol ratio was also kept at $1 / 2$.

\section{Catalytic MB Reduction Study}

The investigation of catalytic reduction study of MB was performed similar to previous work (22). Briefly, a $2 \mathrm{~g} / \mathrm{L}$ of $(6.25 \mathrm{mM})$ stock aqueous MB solution was firstly prepared and the MB reduction studies were conducted by using freshly prepared diluted MB solutions from this stock solution. A $0.32 \mathrm{~g} / \mathrm{L} 1.5 \mathrm{~mL}(1.0 \mathrm{mM})$ of diluted MB solution was mixed with 0.15 mmole $\mathrm{NaBH}_{4}$ containing aqueous solution $(8.5 \mathrm{~mL})$, whose $\mathrm{MB}: \mathrm{NaBH}_{4}$ mole ratio was kept at 1:100 (23). Then, certain amounts of fabric samples in $1 \mathrm{~cm} \times 1 \mathrm{~cm}$ sizes were gently placed in the solutions. After sonicating for $1 \mathrm{~min}$, the changes in the absorbance values of the MB solutions were monitored with UV-Vis spectrophotometry, by taking samples from the solution at certain time intervals and then diluting with water in the ratio of $8 / 1$. The MB reduction yield (\%) was calculated by using Equation (1):

$$
\text { MB reduction }(\%)=\frac{(C o-C)}{C o} * 100
$$


Where Co and $\mathrm{C}$ are the concentrations of $\mathrm{MB}$ solutions at initial and after certain times from catalysis, respectively.

\section{Characterization}

The volume resistivity measurements were performed according to two-probe method using either Thurlby 1503 digital multimeter or Keithley 6517A high resistance meter. For example, while a Keithley 6517A high resistance meter connected to a test fixture was used for the composites with high resistivity values ( $>32$ $\mathrm{M} \square . \mathrm{cm}$ ), for the composites with low resistivity values, the measurement was performed by sandwiching a sample between two stainless steel electrodes that contacted with the electrodes of Thurlby 1503 digital multimeter. The diffraction patterns were recorded with Rigaku Ultima-IV Xray diffraction (XRD) instrument in the $2 \square$ range of $3-90^{\circ}$. Quanta 400F Field Emission SEM instrument was used for the evaluation of surface morphology of the samples after depositing $3 \mathrm{~nm}$ of Au-Pd. A Shimadzu UV-1700 spectrophotometer was used for monitoring the UV-Vis spectra of the dye solutions.

\section{RESULTS AND DISCUSSION}

\section{Effect of Dopant type and dopant concentration}

For the simultaneous POA and $\mathrm{Ag}$ particles deposition on wool fabrics, firstly, the composites were prepared in the medium of $1.0 \mathrm{M} \mathrm{HNO}_{3}$ as we examined before in our previous study (12). However, unlike previous observations, highly conductive (a greenish-black colored) continuous POA deposition could not be obtained on fabric surfaces in the medium of $\mathrm{HNO}_{3}$ and the fabrics were coated locally (Figure S1). When the polymerization medium was selected as sulfonic acids such as PTSA, CSA and DBSA with different side groups that act as anionic dopants for POA polymer $(24,25)$, homogenous and heavy POA and $\mathrm{Ag}$ deposition could be obtained on wool surfaces especially those of DBSA. In DBSA medium, Ag particles could be clearly selected with bare eye. Consequently, the polymerization reactions as well as the investigation parameter were continued in the media of sulfonic acids. In the first view to the composites, no remarkable difference occurred in the polymerizations carried out in PTSA and CSA, when compared to the observations in our previous conductive polymer coating studies. But in the medium of DBSA, it was observed that the appearance of the polymerization solution that wool fabric dipped, was milky due to the formation of 0 anisidine/DBSA complex (25), and its color gradually turned to greenish-black after the addition of oxidant mixtures.

In Figure 1, the effects of type and concentration of selected sulfonic acids on POA/Ag (\%) content and volume resistivity of the composites are given. When the POA/Ag (\%) content values are evaluated in terms of the selected dopant types, it can be seen that the highest POA/Ag (\%) contents were obtained in DBSA medium and the values those of PTSA and CSA were almost close to each other. When the POA/Ag values are examined with concentrations of dopants, it is remarkable that the POA/Ag (\%) content values obtained in all dopant acid solutions gave the same trend and increased up to the certain concentration values. For example, while the values of PTSA increased up to the PTSA concentration of $0.25 \mathrm{M}$ and showed a decrease over this concentration, the values of CSA first increased up to $0.50 \mathrm{M}$ and did not show a significant change with increasing concentration. For the values of DBSA, this increase was also observed up to the concentration value of $0.05 \mathrm{M}$ and then a sharp decrease occurred over this concentration. The highest POA/Ag contents of the composites prepared in different dopant acids increased in the following order: $0.50 \mathrm{M} \mathrm{CSA}$ (POA/Ag: $30 \%$ ) < 0.25 M PTSA (POA/Ag: $38 \%$ ) $<0.05 \mathrm{M}$ DBSA (POA/Ag: $60 \%$ ). The relatively highest $\mathrm{POA} / \mathrm{Ag}(\%)$ value of the composite in DBSA medium could be the result of incorporation of much bulky dopant anion (DBSA) to the POA structure coated on wool surface (26). The decreases obtained at high concentration values may arise from the easy removal of POA/Ag coats from composites with washing processes. Because at higher dopant concentrations, the polymerization of $\mathrm{o}$-anisidine proceeds with more micellar nature and this could lead to the formation of more soluble POA species.

When the resistivity values (Figure $1 \mathrm{~b}$ ) are investigated, it can be understood that the values were not correlated with the values of POA/Ag contents. While the composites obtained in DBSA medium had relatively the highest POA/Ag (\%) contents due to possible high amounts of $\mathrm{Ag}$ particles formation, the resistivity values of the composites in the media of PTSA and CSA were lower than those of DBSA. This observation may suggest that the electrical conductivity phenomenon dominantly based on POA coat of composite than that of $\mathrm{Ag}$ particles (27). When the resistivity values were evaluated with increasing dopant concentrations, it was observed that the values obtained in the media of different acids followed almost similar trend. For example, the volume resistivity values of composites prepared in the media of all acids first decreased to a certain degree and then stayed almost constant or slightly increased. While the resistivity values in PTSA and CSA steadily decreased to the concentration values of $0.50 \mathrm{M}$ and showed a slight increase with increasing concentration, the values of DBSA continued decreasing with increasing DBSA concentration. The lowest resistivity values of the composites were obtained in the ascending order of: $0.50 \mathrm{M}$ PTSA $(75 \mathrm{k} \Omega . \mathrm{cm})<0.50 \mathrm{M}$ CSA $(145 \mathrm{k} \Omega . \mathrm{cm})<$ $0.1 \mathrm{M}$ DBSA $\left(2.38 \times 10^{5} \mathrm{k} \Omega . \mathrm{cm}\right)$. It could be said that this finding emphasized two important points. First, the composite with the highest conductivity was obtained in the medium of PTSA 
although the presence of relatively less amounts of $\mathrm{Ag}$ particles, and second, further increasing of the concentration of the acids did not increase the conductivity but also caused a deterioration.

The doping ability of the selected dopant acids is based on many parameters such as concentration, molecular size and acidity value of the dopant acids, crystallinity and the morphology of the polymer in the literature. For example, Kulkarni et al. reported that the doping ability of PTSA for polyaniline derivatives was related to its ability in the alignment of the polymer chains (24). Because it has been postulated that the polyaniline chains took the form of fibrillary type in the medium of PTSA providing close alignment of the chains, and thus, the conductivity increased by facilitating the transportation of the charges across the polyaniline chains. Another emphasized point is the molecular size of the counter-ion of the dopant acids. With the increase in the length of the counter-ions, it was observed in the literature that the possibility of the expanding the polymer chains increased (28) and after the coupling of these expanded chains, a satisfactory improvement was being recorded in the conductivity (29). One of the most important point that could strongly influence the conductivity is the acidity values of the dopants. The higher the acidity of the dopant acids (low pKa values), the higher the doping level as well as the conductivity of the composites (29). When these values are evaluated according to these above- mentioned parameters, thanks to having relatively the highest acidity value $\left(\mathrm{pK}_{\mathrm{a}}:-2.8\right)$ (30), PTSA may have enable the preparation of composite with relatively the highest conductivity. However, such an approximation is not sufficient in the explanation of the conductivity values of other composites obtained in DBSA and CSA, because the $\mathrm{pK}_{a}$ value of DBSA $(-1.8)$ is lower than that of CSA (1.2). This case may be explained with the morphologic and/or structural difference of the POA polymers coated on wool surfaces in different dopant media. Another possible approximation for the low conductivity values in DBSA medium could be the presence of relatively higher amounts of $\mathrm{Ag}$ particles, causing possible interruptions in the transportation of charges in POA chains. This situation was also encountered in the composites of polyaniline and $\mathrm{Ag}$ particles in the literature (27).
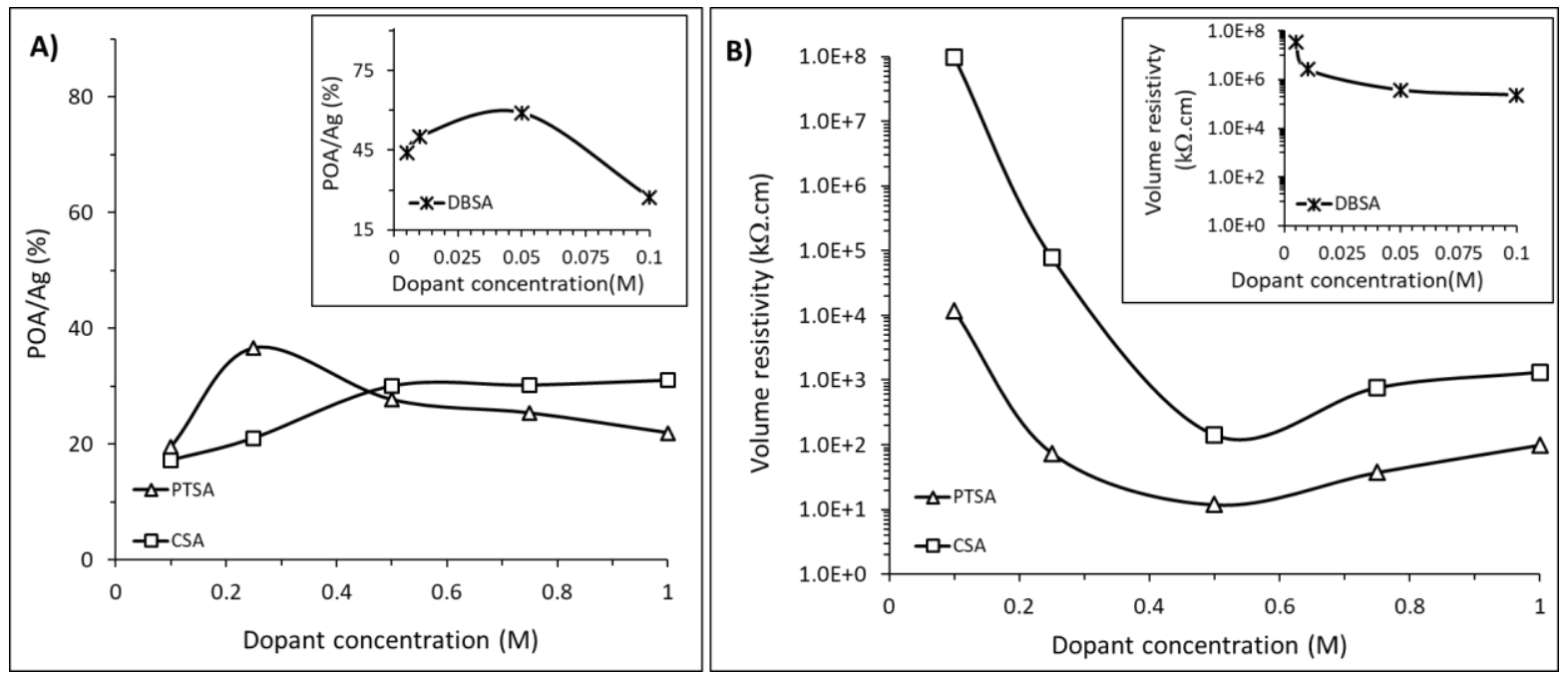

Figure 1: The changes in POA/Ag contents (\%) and volume resistivity of composites with dopant type and concentration (o-anisidine: $0.2 \mathrm{M}$; ammonium persulfate: $0.1 \mathrm{M}$; silver nitrate: $0.1 \mathrm{M}$ )

\section{XRD}

To reveal the crystal structure of the Ag particles that incorporated to the POA/Ag contents of the composites, the diffraction patterns of the composites prepared in different dopant acids media were taken and the comparative results are displayed in Figure 2. In the first view to the diffractograms of all the composites, the characteristic crystalline peaks of metallic $\mathrm{Ag}$ particles can be easily identified at $38^{\circ}, 44^{\circ}, 64^{\circ}$ and $77^{\circ}$ which correspond the (111), (200), (220) and (311) planes of face centered cubic Ag (JCPDS file No. 004-0783). It is also noteworthy that the intensity of these crystalline peaks of metallic Ag particles remarkably differed depending on the dopant acids, and this may suggest that the dominant species also differed in the composites. For example, if the diffractograms are evaluated in terms of the intensity and height values (in cps units) of the main diffraction peak of $\mathrm{Ag}(111)$ at $38^{\circ}$, the composites prepared in different dopant acid media could be listed as: DBSA $(899,1950)>$ CSA $(78,234)>$ PTSA $(79,127)$. This finding supports that DBSA enabled incorporation of high amounts and/or numbers of $\mathrm{Ag}$ particles to the composite surface. It is also remarkable that the composite prepared in CSA medium has different 
diffraction peaks at $32^{\circ}$ and $46^{\circ}$, which may mark the presence of $\mathrm{Ag}_{2} \mathrm{O}$ particles in the planes of (202) and (132) (JCPDS No: 84-1108) (31) that formed during the polymerization. When the crystallite sizes of $\mathrm{Ag}$ particles obtained in different dopant acids media were estimated according to the Scherrer equation, it was observed that the crystallite sizes changed in the following order: $\mathrm{Ag}$ in PTSA $(15.1 \mathrm{~nm})<\mathrm{Ag}$ in DBSA $(23.8 \mathrm{~nm})<\mathrm{Ag}$ in CSA $(44.7 \mathrm{~nm})$.
Apart from the crystalline peaks, the characteristic peaks of conductive POA polymer around $7^{\circ}$ and $25^{\circ}$ (32) could only be clearly detected in the diffractogram of the composite in PTSA medium. But these peaks belonging to the composites obtained in the media of CSA and DBSA could not be separately identified due to the superimposition with the peaks of wool fabric around $9^{\circ}, 20^{\circ}(33)$.

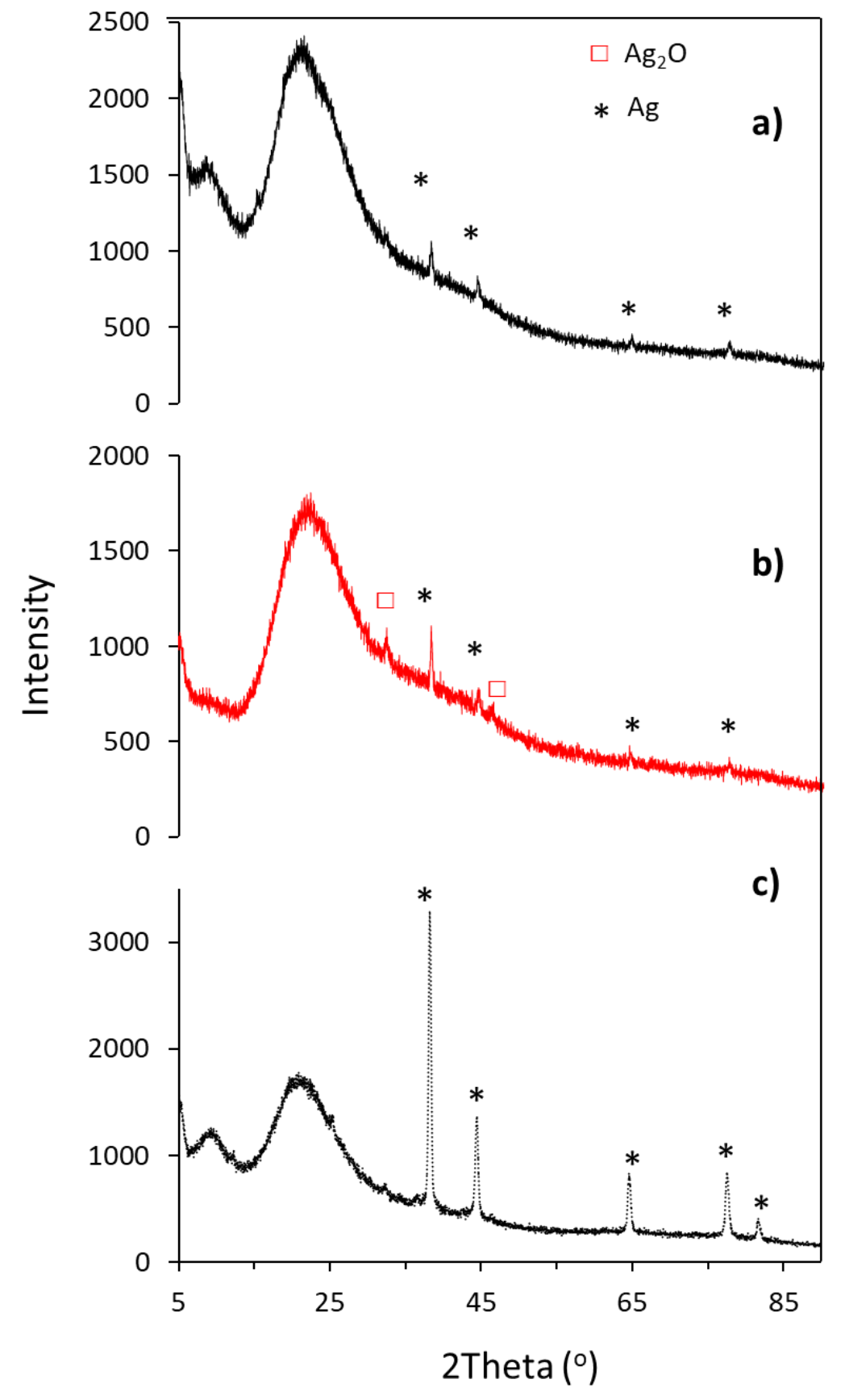

Figure 2: XRD diffraction patterns of the samples obtained in the media of a) $0.50 \mathrm{M}$ PTSA (POA/Ag content: $38 \%$, b) 0.50 M CSA (POA/Ag content: $30 \%$ ) and c) 0.05 M DBSA (POA/Ag content: $60 \%$ ).

\section{SEM}

SEM micrographs of the composites prepared in the media of different dopant acids are displayed in Figure 3. When all the micrographs are first examined in terms of the deposition, it can be noticed that the wool fabric were homogenously and intensively covered with POA polymers in different morphologies/structures from adherent globular structures to mud-like irregular aggregates with changing dopant acids. For example, the POA coats prepared in PTSA and CSA media have similar appearances as adherent 
globules-like structures (Figure $3 a-f)$. This morphology of the polymer gives the impression of POA polymers were first occurred on the whole surface of wool fabric as nucleation even for the scale regions; then the growth/construction of the polymer progressed on the coated sites leading to the formation of top-up arrangement of POA. However, in the medium of DBSA (Figure $3 \mathrm{~g}, \mathrm{i})$, the surface of the wool was covered with differently, with a morphology of mud-like irregular shaped aggregates especially formed from overlapped polymer layers. It can also be said that the surface of the composite prepared in DBSA medium was relatively smoother than those of PTSA and CSA. If the micrographs are evaluated in terms of the Ag particles, images show that the shapes of Ag particles in the media of all dopant acids have similar structures, separately interspersed shiny regular geometric shapes such as rectangular, square and ellipse, which can be easily selected in the micrographs at higher magnifications (Figure $3 c, f, i)$. However, their distribution and homogeneity were quite differentiated depending on the acids. In PTSA medium, while very few amounts of separate Ag particles were localized on POA coat, in CSA, it was observed that the separate $\mathrm{Ag}$ particles formed an adherent heap-like structure on POA. Unlike from the media of PTSA and CSA, in DBSA, it can be clearly visualized that the Ag particles were homogenously and intensively present on the POA coated-surfaces without any aggregation signs as compatible with the literature (34). The micrograph of the composite in DBSA medium also gives the impression of DBSA distinguished the Ag particles by covering their surroundings. This observation suggests that DBSA played a rather effective role in the stabilizing of $\mathrm{Ag}$ particles during the polymerization. However, particle sizes of the $\mathrm{Ag}$ particles obtained in the media PTSA, CSA and DBSA could not be precisely determined from SEM images but it could be estimated that their sizes are quite compatible with the findings of the XRD measurements.
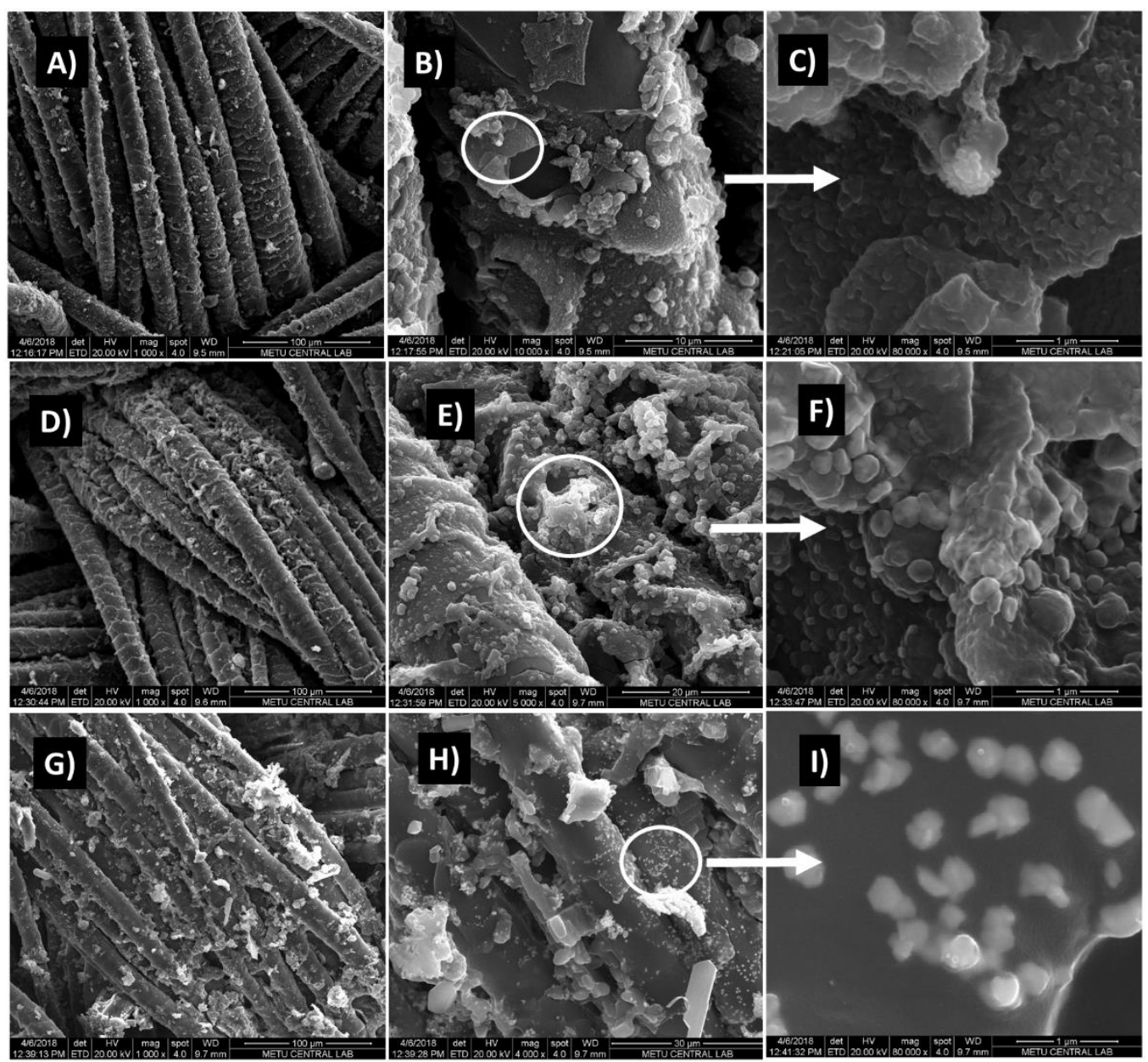

Figure 3: SEM micrographs of the composites obtained in the media of a), b), c) $0.50 \mathrm{M}$ PTSA (POA/Ag content: $38 \%), d), e)$, f) $0.50 \mathrm{M} \mathrm{CSA}(\mathrm{POA} / \mathrm{Ag}$ content: $30 \%$ ) and g), h), i) $0.05 \mathrm{M} \mathrm{DBSA}(\mathrm{POA} / \mathrm{Ag}$ content: $60 \%)$.

\section{EDX}

To determine the amount of $\mathrm{Ag}$ particles that contributed to the POA/Ag contents of the composites semi-quantitatively, the EDX spectra of the composites prepared in the media of different acids were taken from the backscattering SEM micrographs, and the results are represented in Figure 4 . When the figure is generally examined, firstly it is noteworthy that the composite prepared in DBSA medium had 
relatively the highest $\mathrm{Ag}$ signals and thus $\mathrm{Ag}$ particles content (Figure 4c). The Ag particles content of other composites in PTSA and CSA were significantly lower than that of DBSA, and this can also be understood from the less number of $\mathrm{Ag}$ particles obtained in the relevant micrographs (Figure $3 b, e, h$ ).

To estimate the relative doping degree of the composites semi-quantitatively, the atomic percentage values of $S$ and $C$ in EDX spectra were proportioned as S/C. It was observed that the S/C values were in the following ascending order: DBSA $\quad(\sim 1 / 13) \quad(0.078)<C S A \quad(\sim 1 / 9) \quad(0.106)$ $<$ PTSA $(\sim 1 / 9)(0.108)$. If these values are evaluated considering the molecular weights of the dopant acids, while the value of $1 / 9$ of PTSA $\left(\mathrm{C}_{7} \mathrm{H}_{8} \mathrm{O}_{3} \mathrm{~S} . \mathrm{H}_{2} \mathrm{O}\right)$ is lower than the actual $1 / 7$ value of $\mathrm{S} / \mathrm{C}$ in PTSA, this value is quite close to the actual $1 / 12$ value of DBSA $\left(\mathrm{C}_{12} \mathrm{H}_{30} \mathrm{O}_{3} \mathrm{~S}\right)$ and higher than the actual value of $1 / 10$ of CSA $\left(\mathrm{C}_{10} \mathrm{H}_{16} \mathrm{O}_{4} \mathrm{~S}\right)$.

According to this, it could be interpreted that despite the presence of $\mathrm{C}$ atoms in POA polymer, by having higher S/C atomic ratio, namely receiving intensive $S$ signals from the composites in CSA and DBSA may mark the presence of higher numbers of dopant molecules than that of POA chains. This may also point out shorter POA chains coated the surface of the composites in the media of DBSA and CSA, but the amounts of dopant molecules that correspond the unit area of the polymer may be intensive. In other words, the dopant molecules may have intensively placed between POA chains. Unlike DBSA and CSA, having lower $S / C$ atomic ratio of the composite in PTSA may suggest the presence of longer POA chains with relatively low numbers of dopant molecules, due to the possible steric hindrance of PTSA.
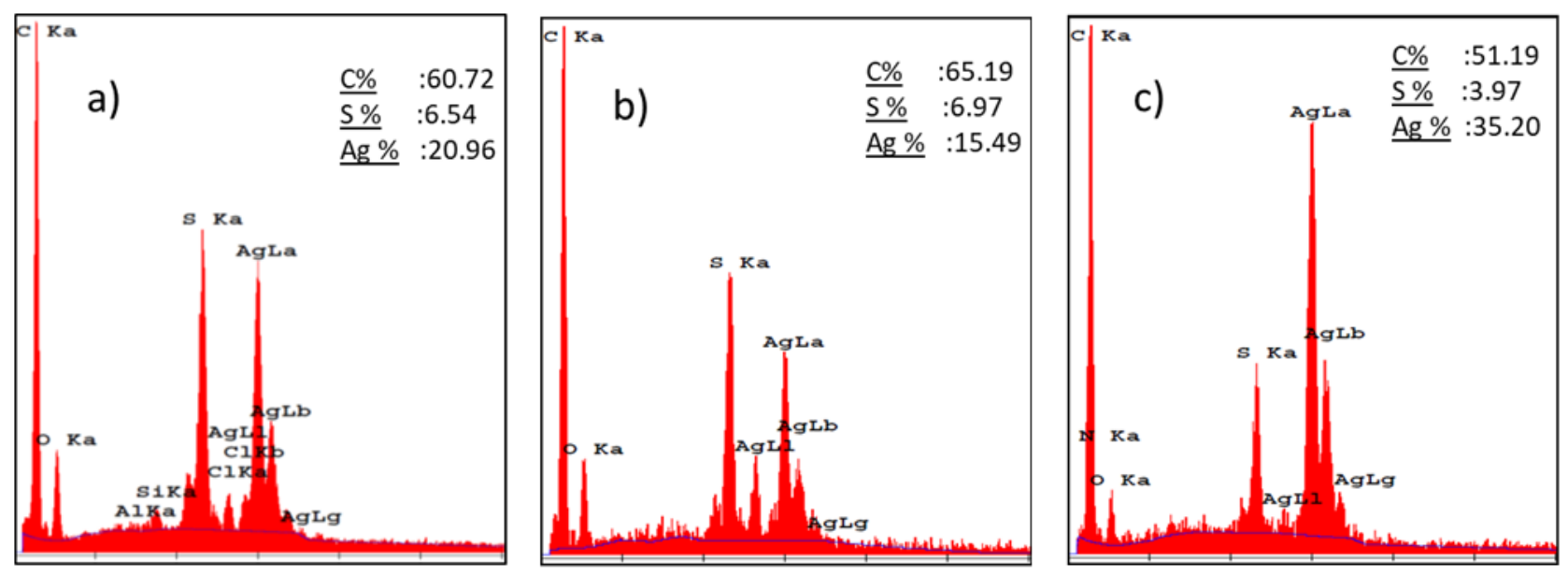

Figure 4: EDX spectra taken from backscattering SEM micrographs of the composites obtained in the media of a) $0.50 \mathrm{M}$ PTSA (POA/Ag content: $38 \%$ ), b) $0.50 \mathrm{M} \mathrm{CSA}$ (POA/Ag content: $30 \%$ ) and c) $0.05 \mathrm{M}$ DBSA (POA/Ag content: $60 \%$ ).

\section{Catalytic performances in MB reduction}

To monitor the usability of $\mathrm{Ag}$ particles that contributed to the composites as simple catalyst, the composite samples were examined in the catalytic reduction of MB dye to $L M B$ in presence of excess $\mathrm{NaBH}_{4}$ reducing agent. The monitoring the reduction of $\mathrm{MB}$ (blue color) to LMB (transparent) is known to be a facile method in the literature for the determination of the catalytic performance of a metallic nanoparticle $(23,35-37)$. For this purpose, the UV-Vis spectra of MB solutions were taken in presence and absence of the composites and derivative results from these spectra are illustrated in the range of Figure 5-7. 


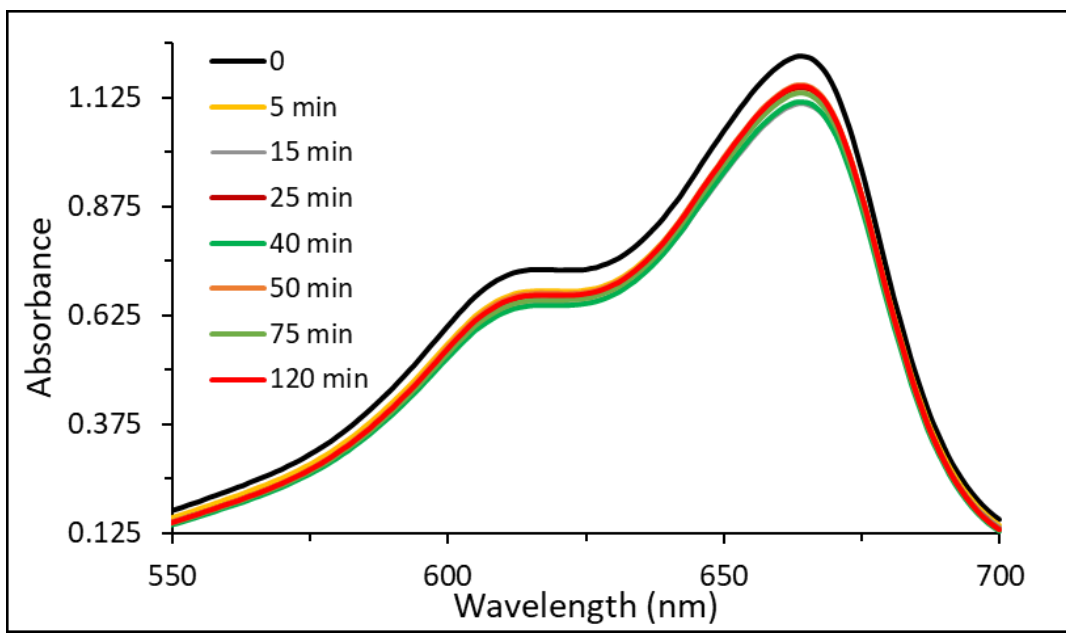

Figure 5: Time dependent UV-Vis absorbance spectra of MB solution catalytically reduced to LMB using $\mathrm{NaBH}_{4}$ in the absence of composites.

As can be seen from Figure 5, the UV-Vis absorption band of aqueous MB solution (664 $\mathrm{nm})$, corresponding $\mathrm{n}-\pi^{*}$ transition, slightly decreased with time and this showed that the reduction of $M B$ scarcely occurred in the absence of composites. This could also be determined from very weak decolorization of MB solution. When a composite sample was introduced to the solution, it was observed that the surface of the composite covered with gas bubbles, the color of the dye solution gradually turned to light blue to transparent, and consequently, the absorbance values of the MB solutions markedly decreased with time (Figure 6a-c). According to the MB reduction (\%) values derivative from UV-Vis spectra of the relevant composites, the percent reduction values of the composites prepared in different acids media steadily increased with time, reached a maximum and stayed almost constant, and the values changed depending on the selected dopant acids. The highest catalytic activity ( $\sim 70 \%)$ of the composite was obtained as 75 min in DBSA medium (Figure 6c). The MB percent reduction values of other composites prepared in PTSA and CSA were almost close to each other and obtained around $\sim 50 \%$ and $\sim 60 \%$, respectively.
It has been reported that the decolorization of dyes through a catalytic reduction reaction using metal nanoparticles in presence of a reducing agent such as $\mathrm{NaBH}_{4}$ takes place by relaying metal nanoparticles the electrons from $\mathrm{BH}_{4}{ }^{-}$ anions to the acceptor dye molecules. $(36,38)$. The catalytic activity performance of a metal nanoparticle could also be related with its particle size, surface area and distribution frequency and/or its loading concentration $(39,40)$. If the MB reduction capabilities of the composites obtained in different media are evaluated considering these parameters, since enabling homogenous and intensive $\mathrm{Ag}$ particles distribution and thus giving the highest $\mathrm{Ag}$ particles contribution to the POA/Ag contents of the composite, it could be said that DBSA is the most suitable medium to obtain high $M B$ reduction values. However, when this maximum value is compared with the values present in the literature $(22,35)$, its performance could be graded as moderate, and since the Ag particles might have located under and/or between the POA-DBSA coating and this may possibly limit the transportation of electrons via Ag particles. 

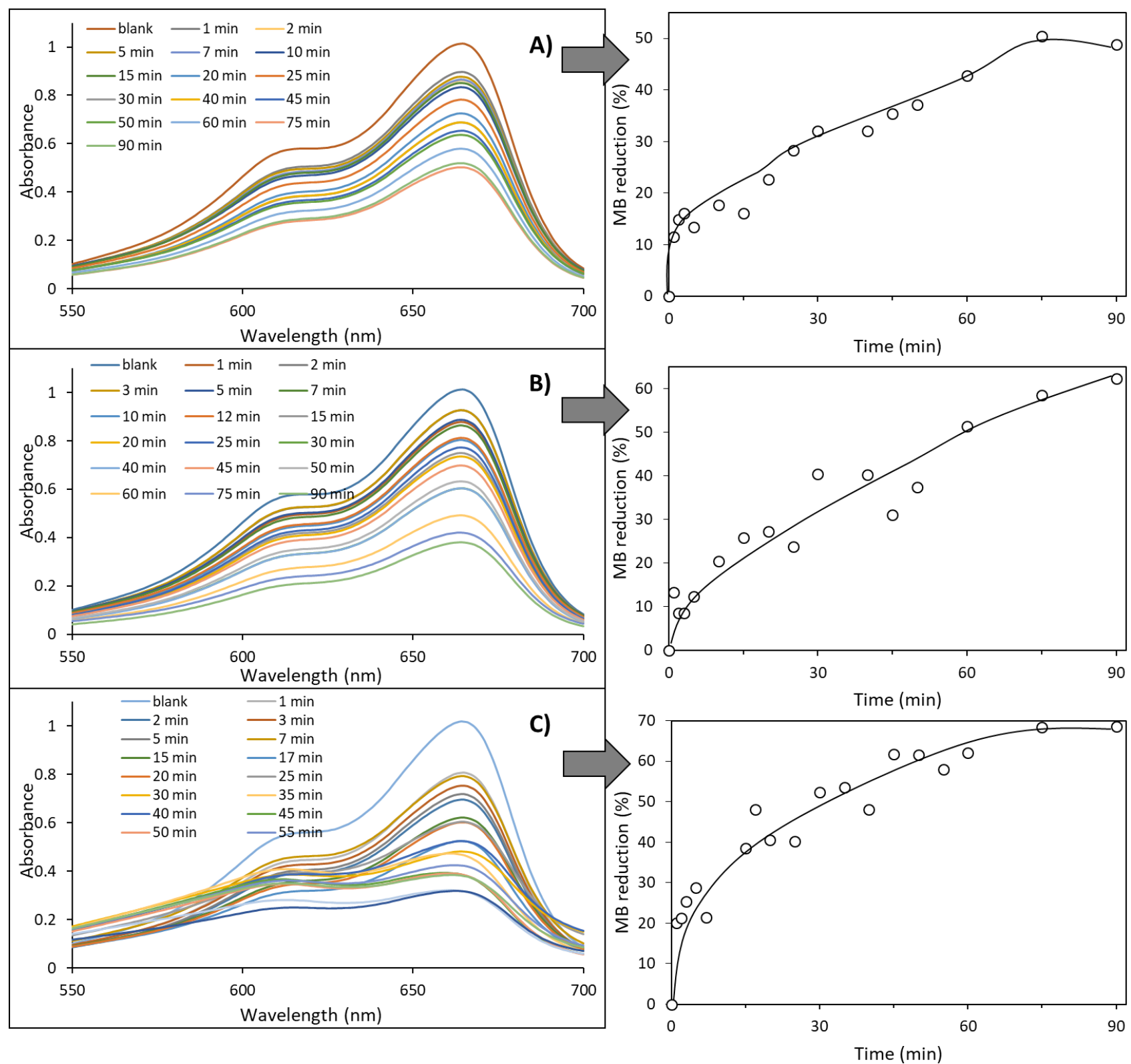

Figure 6: Time dependent UV-Vis absorbance spectra of MB solutions catalytically reduced to LMB using Ag particles and POA deposited wool fabrics prepared in a) PTSA, b) CSA c) DBSA (The graphs across the arrows show the change in MB reduction (\%) values of the relevant composites) ( $[\mathrm{PTSA}]: 0.50 \mathrm{M}$, [CSA]: $0.50 \mathrm{M},[\mathrm{DBSA}]: 0.01 \mathrm{M})$.

The effects of the contribution of POA prepared in different dopant acids media to the catalytic activity were also tested in the reduction of MB, and the data obtained are given in Figure 7. It can be seen that the MB reduction (\%) values of all the composites were remarkably lower than the composites containing Ag particles. For example, the percent $\mathrm{MB}$ reduction values of the composite in PTSA were quite irregular and low. The values of CSA were relatively higher than the values obtained in the media of DBSA and PTSA and this may suggest an undeniable catalytic activity in $M B$ reduction. Nevertheless, the values did not show a consistent trend and remarkably decreased after $30 \mathrm{~min}$. 

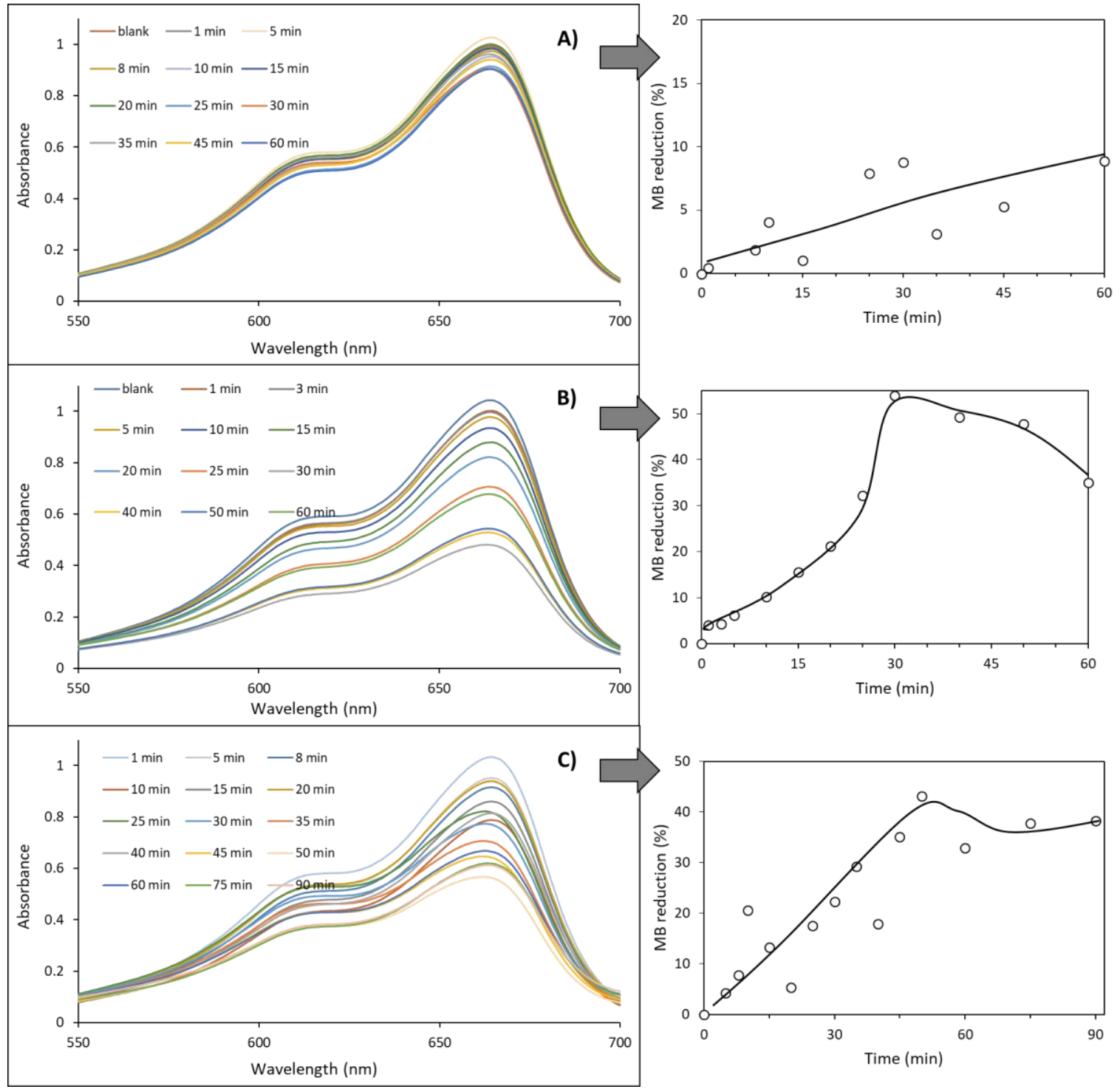

Figure 7: Time dependent UV-Vis absorbance spectra of MB, catalytically reduced to LMB using POA coated wool fabrics in absence of Ag particles prepared in a) PTSA b) CSA and c) DBSA (The graphs across the arrows show the change in MB reduction (\%) values of the relevant composites) ([PTSA]:

$0.50 \mathrm{M}$, [CSA]: $0.50 \mathrm{M}$, [DBSA]: $0.01 \mathrm{M}$ ).

\section{CONCLUSION}

It was shown with present study that the surface modification of wool fabrics could be achieved by coating with one of the most conductive polyaniline derivatives of POA and metallic Ag particles, through one-step oxidative polymerization technique. It was observed that the selection of dopant acids (especially sulfonic acids) as polymerization medium was crucial for continuous and homogenous POA/Ag deposition on wool surfaces. Despite giving the lowest conductivity values, DBSA enabled the contribution of relatively, the highest contents of POA/Ag to the composite, among in other sulfonic acids. XRD results suggested the presence of noble Ag particles on all the composites prepared in different acids media, but in CSA medium, the formation of $\mathrm{Ag}_{2} \mathrm{O}$ was also detected. SEM micrographs revealed that the morphology of the conductive POA coat on wool fabrics changed depending on the dopant acids ranging from adherent globules-like structures to mud-like irregular aggregates. It was also observed from SEM micrographs that, although the shapes of $\mathrm{Ag}$ particles in the media of all dopant acids were almost similar, the distribution and homogeneity of $\mathrm{Ag}$ particles were remarkably differed depending on the selected dopant acids. The EDX results indicated that the contribution of dopant molecules were relatively high in the media of CSA and DBSA. The results of catalytic performance of the composites in MB reduction concluded that despite the presence of high amounts of conductive POA and $\mathrm{Ag}$ particles coated on wool fabrics, the possible location of Ag particles might limit the performance. One of the most significant results of this study was the moderate catalytic performance of the composites in DBSA medium. 


\section{REFERENCES}

1. Erdoğan $M K$, Karakişla $M$, Saçak $M$. Preparation, Characterization and Electromagnetic Shielding Effectiveness of Conductive Polythiophene/Poly(ethylene terephthalate) Composite Fibers. Journal of Macromolecular Science, Part A. $2012 ; 49(6): 473-82$.

2. Saini P, Choudhary V. Conducting polymer coated textile based multilayered shields for suppression of microwave radiations in 8.2-12.4 $\mathrm{GHz}$ range. Journal of Applied Polymer Science. 2013;129(5):2832-9.

3. Saini $P$, Choudhary V, Dhawan SK. Improved microwave absorption and electrostatic charge dissipation efficiencies of conducting polymer grafted fabrics prepared via in situ polymerization. Polymers for Advanced Technologies. 2012;23(3):343-9.

4. Bhat NV, Seshadri DT, Nate MM, Gore AV. Development of conductive cotton fabrics for heating devices. Journal of Applied Polymer Science. 2006;102(5):4690-5.

5. Oh KW, Park HJ, Kim SH. Stretchable conductive fabric for electrotherapy. Journal of Applied Polymer Science. 2003;88(5):1225-9.

6. Li Y, Cheng XY, Leung MY, Tsang J, Tao XM, Yuen MCW. A flexible strain sensor from polypyrrole-coated fabrics. Synthetic Metals. 2005;155(1):89-94.

7. Acar H, Karakisla M, Sacak M. Potassium persulfate-mediated preparation of conducting polypyrrole/polyacrylonitrile composite fibers: Humidity and temperature-sensing properties. Journal of Applied Polymer Science. 2012;125(5):3977-85.

8. Hamedi M, Forchheimer R, Inganäs O. Towards woven logic from organic electronic fibres. Nature Materials. 2007;6:357.

9. Kelly FM, Meunier L, Cochrane C, Koncar V. Evaluation of Solid or Liquid Phase Conducting Polymers Within a Flexible Textile Electrochromic Device. Display Technology, Journal of. 2013;9(8):626-31.

10. Erdoğan MK, Karakışla M, Saçak M. Polypyrrole and silver particles coated poly(ethylene terephthalate) nonwoven composite for electromagnetic interference shielding. Journal of Composite Materials.0(0):0021998317724859.

11. Firoz Babu K, Dhandapani P, Maruthamuthu S, Anbu Kulandainathan M. One pot synthesis of polypyrrole silver nanocomposite on cotton fabrics for multifunctional property. Carbohydrate Polymers. 2012;90(4):1557-63.
12. Erdoğan $M K$, Karakışla $M$, Saçak $M$. Fabrication of poly(o-Anisidine)/Ag particles coated poly(ethylene terephthalate) nonwoven composite and investigation of antibacterial activity. Polymer Composites.0(0).

13. Mondal S, Rana U, Malik S. Facile Decoration of Polyaniline Fiber with Ag Nanoparticles for Recyclable SERS Substrate. ACS Applied Materials \& Interfaces. 2015;7(19):10457-65.

14. $\mathrm{Bu} \mathrm{Y,} \mathrm{Chen} \mathrm{Z}$. Role of Polyaniline on the Photocatalytic Degradation and Stability Performance of the Polyaniline/Silver/Silver Phosphate Composite under Visible Light. ACS Applied Materials \& Interfaces. 2014;6(20):17589-98.

15. Blinova NV, Stejskal J, Trchová M, Sapurina I, Ćirić-Marjanović $\mathrm{G}$. The oxidation of aniline with silver nitrate to polyaniline-silver composites. Polymer. 2009;50(1):50-6.

16. Bober $P$, Stejskal J, Trchová M, Hromádková J, Prokeš J. Polyaniline-coated silver nanowires. Reactive and Functional Polymers. $2010 ; 70(9): 656-62$.

17. Blinova NV, Bober P, Hromádková J, Trchová M, Stejskal J, Prokeš J. Polyaniline-silver composites prepared by the oxidation of aniline with silver nitrate in acetic acid solutions. Polymer International. 2010;59(4):437-46.

18. Attia MF, Azib T, Salmi Z, Singh A, Decorse $P$, Battaglini $N$, et al. One-step UV-induced modification of cellulose fabrics by polypyrrole/silver nanocomposite films. Journal of Colloid and Interface Science. 2013;393:130-7.

19. Gashti M, Ghehi S, Arekhloo S, Mirsmaeeli A, Kiumarsi A. Electromagnetic shielding response of UV-induced polypyrrole/silver coated wool. Fibers Polym. 2015;16(3):585-92.

20. Bober P, Stejskal J, Trchová M, Prokeš J. Polyaniline-silver composites prepared by the oxidation of aniline with mixed oxidants, silver nitrate and ammonium peroxydisulfate: The control of silver content. Polymer. 2011;52(26):5947-52.

21. Bober P, Stejskal J, Trchová M, Prokeš J, Sapurina I. Oxidation of Aniline with Silver Nitrate Accelerated by p-Phenylenediamine: A New Route to Conducting Composites. Macromolecules. 2010;43(24):10406-13.

22. Erdoğan $M K$, Karakışla $M$, Saçak $M$. Morphologically different silver particles decorated- conductive poly(o-anisidine)/wool fabric composites and investigation of catalytic activity in reduction of methylene blue. Materials Chemistry and Physics. 2019;225:72-83. 
23. Vidhu VK, Philip D. Catalytic degradation of organic dyes using biosynthesized silver nanoparticles. Micron. 2014;56:54-62.

24. Kulkarni MV, Viswanath AK, Khanna PK. Synthesis and characterization of poly(N-methyl aniline) doped with sulphonic acids: Their application as humidity sensors. Journal of Applied Polymer Science. 2006;99(3):812-20.

25. Babazadeh M. Aqueous dispersions of DBSAdoped polyaniline: One-pot preparation, characterization, and properties study. Journal of Applied Polymer Science. 2009;113(6):3980-4.

26. Ichinohe $D$, Aral $T$, Kise $H$. Synthesis of soluble polyaniline in reversed micellar systems. Synthetic Metals. 1997;84(1-3):75-6.

27. Bober $P$, Trchová $M$, Prokeš J, Varga $M$, Stejskal J. Polyaniline-silver composites prepared by the oxidation of aniline with silver nitrate in solutions of sulfonic acids. Electrochimica Acta. 2011;56(10):3580-5.

28. Cao $Y$, Smith $P$, Heeger AJ. Counter-ion induced processibility of conducting polyaniline and of conducting polyblends of polyaniline in bulk polymers. Synthetic Metals. 1992;48(1):917.

29. Long Y, Chen Z, Wang N, Zhang Z, Wan M. Resistivity study of polyaniline doped with protonic acids. Physica B: Condensed Matter. 2003;325:208-13.

30. Guthrie JP. Hydrolysis of esters of oxy acids: pKa values for strong acids; Brønsted relationship for attack of water at methyl; free energies of hydrolysis of esters of oxy acids; and a linear relationship between free energy of hydrolysis and pKa holding over a range of 20 pK units. Canadian Journal of Chemistry. 1978;56(17):2342-54.

31. Yang $H$, Ren $Y-y$, Wang $T$, Wang $C$. Preparation and antibacterial activities of $\mathrm{Ag} / \mathrm{Ag}+/ \mathrm{Ag} 3+$ nanoparticle composites made by pomegranate (Punica granatum) rind extract. Results in Physics. 2016;6:299-304.

32. Patil D, Patil P, Seo Y-K, Hwang YK.
Poly(o-anisidine)-tin oxide nanocomposite: Synthesis, characterization and application to humidity sensing. Sensors and Actuators B: Chemical. 2010;148(1):41-8.

33. Lovejoy KS, Lou AJ, Davis LE, Sanchez TC, Iyer S, Corley CA, et al. Single-Pot ExtractionAnalysis of Dyed Wool Fibers with Ionic Liquids. Analytical Chemistry. 2012;84(21):9169-75.

34. Dhibar S, Das CK. Silver Nanoparticles Decorated Polyaniline/Multiwalled Carbon Nanotubes Nanocomposite for High-Performance Supercapacitor Electrode. Industrial \& Engineering Chemistry Research. 2014;53(9):3495-508.

35. Nadagouda MN, Desai I, Cruz C, Yang DJ. Novel Pd based catalyst for the removal of organic and emerging contaminants. RSC Advances. 2012;2(19):7540-8.

36. Ganapuram BR, Alle M, Dadigala R, Dasari A, Maragoni V, Guttena V. Catalytic reduction of methylene blue and Congo red dyes using green synthesized gold nanoparticles capped by salmalia malabarica gum. International Nano Letters. $2015 ; 5(4): 215-22$.

37. Kariuki VM, Yazgan I, Akgul A, Kowal A, Parlinska M, Sadik OA. Synthesis and catalytic, antimicrobial and cytotoxicity evaluation of gold and silver nanoparticles using biodegradable, [capital Pi]-conjugated polyamic acid. Environmental Science: Nano. 2015;2(5):51827.

38. Gupta N, Singh HP, Sharma RK. Metal nanoparticles with high catalytic activity in degradation of methyl orange: An electron relay effect. Journal of Molecular Catalysis A: Chemical. 2011;335(1):248-52.

39. Zielińska-Jurek A, Wei Z, Wysocka I, Szweda $P$, Kowalska $E$. The effect of nanoparticles size on photocatalytic and antimicrobial properties of $\mathrm{Ag}$ $\mathrm{Pt} / \mathrm{TiO} 2$ photocatalysts. Applied Surface Science. 2015;353:317-25.

40. Height MJ, Pratsinis SE, Mekasuwandumrong $\mathrm{O}$, Praserthdam P. Ag-ZnO catalysts for UVphotodegradation of methylene blue. Applied Catalysis B: Environmental. 2006;63(3):305-12. 